\title{
Le asimmetrie delle libertà del lavoro (al plurale)
}

\author{
di Laura Cerasi
}

Asymmetries in the liberties (plural) of labour

This reply deals with the arguments developed by the previous discussants with reference to the non-linear view of the relationship between labour history and Marxism and to the character of a crossroads between State and society, private and public, individual and coactive, inherent in the dimension of labour; both views are considered useful for the advancement of studies.

Key words: Labour, Liberty, marxism, State, conflict

Desidero innanzitutto ringraziare la direzione di "Società e Storia" per aver promosso e ospitato la discussione sul secondo Quaderno delle edizioni della Società Italiana di Storia del Lavoro (SISLav) da me curato, Le libertà del lavoro. Storia, diritti, società; desidero ringraziare in particolare i colleghi - Irene Stolzi, Andrea Lanza, Paolo Mattera - che hanno accettato di parteciparvi. I loro commenti permettono infatti di ripensare al progetto di cui il volume è espressione e misurarne, a distanza di un breve lasso temporale, la riuscita. Il fatto che tutti abbiano colto il taglio multidisciplinare del lavoro, e ne abbiano ricavato motivo di apprezzamento, è un primo segnale importante che l'impegno messo in campo dalla SISLav, la cui ragione sociale è in primo luogo quella di federare gli studiosi che da angolature disciplinari diverse si occupano del lavoro in prospettiva storica, ha una sua ragione d'essere ed intercetta un'esigenza sentita. Il fatto, inoltre, che da tutti sia stato posto in evidenza come i contributi raccolti si misurino, ciascuno nel proprio campo (dalla business history alla storia del diritto, alla storia delle istituzioni, alla storia della cultura e dei concetti) con lo stato degli studi - talvolta, come nel contributo di Maria Grazia Meriggi, anche in ampia prospettiva temporale offrendo un saggio di storia della storiografia - e su questa base vogliano individuare nuovi problemi di ricerca e indicare linee di sviluppo improntate in varia misura al superamento di paradigmi consolidati, è conferma dell'opportunità della formula adottata dalla SISLav per il suo primo congresso, dal quale sono stato ricavati e rielaborati i saggi presentati nel volume. Sulle linee di sviluppo individuate in quell'occasione sono stati infatti impostati i cantieri della Società, che in più di un caso danno corpo ad una nuova storia del lavoro nel senso indicato da Mattera, in direzione di una global labour history che attraversi non solo le partizioni fra le aree geografiche

Laura Cerasi è ricercatore confermato in Storia Contemporanea presso l'Universià di Venezia - laura.cerasi@unive.it.

Società e storia n. 163 2019, Issn 0391-6987, Issn-e 1972-5515 
ma anche quelle fra le epoche e le periodizzazioni, come è testimoniato dal recente volume delle edizioni SISLav curato da Giulia Bonazza e Giulio Ongaro ${ }^{1}$, o dalla prima conferenza dell'European Labour History Network organizzata a Torino dalla Società nel dicembre $2015^{2}$.

Fra gli spunti di riflessione critica offerti dai commenti, vorrei soffermarmi su quello più radicale, avanzato da Andrea Lanza, che pone in evidenza l'assenza nel volume di un rifermento al marxismo, come riflesso della medesima assenza nel dibattito storiografico degli ultimi anni sul tema del lavoro, che vede il marxismo "sfumare, quasi fosse inconsistente patina ideologica e non modello interpretativo". L'osservazione è importante, perché sottolinea un dato innegabile, che forse ora proprio per il nuovo dinamismo manifestato dalla storia del lavoro può essere visto sotto una luce diversa. È vero, infatti, che in particolare nel caso italiano l'impronta del marxismo ha segnato tanto le forme di organizzazione del movimento operaio, a partire dal momento in cui entravano nella dimensione politica ${ }^{3}$, quanto la riflessione storiografica, a partire da quell" "incontro con il marxismo" che si trova all'origine del rinnovamento degli studi storici del primo Novecento ${ }^{4}$. Un incontro che implicava già allora l'interrogativo, richiamato da Lanza, sulla natura del marxismo come dottrina o come metodo, come disvelamento dei rapporti sociali ed economici nella contemporaneità del capitalismo ma anche nella storia, o come invito, nell'interpretarle, alla valorizzazione della dimensione economica e sociale. E se Benedetto Croce, come noto, si era attestato sulla riduzione del marxismo a metodo, i giovani storici come Gaetano Salvemini, Gioacchino Volpe, Gino Arias pur in misura diversa si spingevano ad assumere la dinamica conflittuale fra le classi come motore della storia ${ }^{5}$. Si trattava di una storiografia, non a caso denominata economico-giuridica, che introduceva come motivo di innovazione il nesso tra l'elemento economico e quello istituzionale, e che risentiva in qualche caso delle questioni del tempo, come il tumultuoso sviluppo del movimento socialista e sindacale, nell' attenzione riservata alle forme organizzative delle classi popolari. Va però osservato che negli esponenti che hanno dato il contributo più incisivo e duraturo, come Gino Luzzatto, tale prospettiva ha poi assunto la forma di vera e propria storia economica, e come tale è stata a lungo ed egregiamente praticata; mentre chi ha posto il lavoro, in quanto tale, come nucleo vitale delle trasformazioni storiche è stato Gioacchino Volpe. Era un "lavoro" espressione delle forze creative e produttive del popolo, cui veniva tanto più dato rilievo quanto più tanto più la prospettiva dello storico abruzzese si andava condensando nel vitalismo nazionalista che ha poi caratterizzato la sua storiografia ${ }^{6}$.

In questo senso, almeno nel caso italiano va rilevata un'asimmetria originaria tra marxismo e storia del lavoro; un' asimmetria che a tratti emerge con maggiore chiarezza. È noto come il primo grande progetto scientifico ed editoriale di una storia del lavoro che attraversasse le epoche storiche e giungesse alla contemporaneità è stato concepito in epoca tardofascista dal bottaiano Riccardo Del Giudice: la cornice corporativa forniva l'orizzonte di senso in cui situare i fenomeni di organizzazione e rappresentanza del lavoro nella storia, e il primo volume uscito, firmato da Amintore Fanfani, ne era

1. Bonazza, Ongaro (2018).

2. Si veda il programma: http://www.storialavoro.it/turin-2015/.

3. Favilli, (1996); Id. (2006).

4. Si veda ancora, in questa prospettiva, Cervelli (1983).

5. Artifoni (1990).

6. Cervelli (1977), p. 51. 
espressione ${ }^{7}$. Il secondo sarebbe uscito nel 1944 ad opera di Luigi Dal Pane, e andrebbe forse ancora approfondito, al di là delle convenzionali cesure, quanto dovesse a questo motivo originario la successiva opera storiografica di Dal Pane, ispirata ad una concezione materialistica della storia che intrecciava storia economica e storia del lavoro, quest'ultima oggetto di speciali riflessioni di metodo con La storia come storia del lavoro (1968). Con Dal Pane e non solo, marxismo e lavoro si saldavano in una storiografia economica e sociale che tuttavia tendeva a rappresentare il lavoro non tanto come oggetto di indagine in sé stesso, ma attraverso le sue forme politiche e organizzative.

E se è vero come ricorda Lanza che con E.P. Thompson già dalla fine degli anni sessanta il rinnovamento degli studi sul lavoro ha preso il via dall'interno del campo marxi$\mathrm{sta}^{8}$, è stato anche osservato come la sconfitta del movimento operaio negli anni ottanta abbia "paradossalmente" liberato energie per un ampliamento dell'orizzonte degli studi sul lavoro, comprensivo di terreni trascurati dalle fasi anteriori e più "militanti" della storiografia: in un dialogo più stretto con le scienze sociali e antropologiche, sono stati messi a fuoco i comportamenti individuali, la dimensione comunitaria, la costruzione di identità e di reti di relazioni sociali ${ }^{9}$. Nel caso italiano soprattutto, ad evitare il rischio di "ghettizzazione" cui la storia del lavoro sembrava destinata a seguito dalla caduta delle tensioni etico-politiche alimentate dalla militanza è stata decisiva la transizione, a partire dalla metà degli anni settanta, della storia del movimento operaio dal dominio della storia politica e dei gruppi dirigenti, a quello della storia sociale, come parte integrante delle sue trasformazioni ${ }^{10}$. Per entrare anche, in qualche misura, nell' ambito della storia culturale, con il rischio di schiacciamento della dimensione del conflitto che può esserne il portato.

Il richiamo rivolto alla nuova stagione di studi sul lavoro a fare i conti con il marxismo è perciò opportuno; va accolto come indicazione di una linea di ripensamento critico che vada oltre l'arricchimento disciplinare che pure ha contribuito a far "uscire dal ghetto" la storia del lavoro e a renderla in grado di misurarsi con le grandi questioni che attraversano la nostra contemporaneità su scala globale, e come invito a cogliere il residuo insolubile dei rapporti di lavoro - come mostrano ad esempio le riflessioni di Francesca Coin ${ }^{11}$. E altrettanto fecondo può essere il suggerimento a ripensare la dimensione del conflitto non tanto in termini antagonistici quanto come produttore di identità e come condizione della ricomposizione sociale attraverso la definizione di regole e istituzioni.

Da questo punto di vista, si potrebbe osservare che in effetti in questo volume la dimensione contrastiva o conflittuale è stata affidata soprattutto alla riflessione sul diritto e le istituzioni, sul sistema normativo cioè entro il quale i rapporti di lavoro hanno preso forma. È una prospettiva che emerge anche dalle stimolanti osservazioni di Irene Stolzi, che mi pare non solo abbiano colto magistralmente l'intento complessivo che attraversa i contributi del volume, ma aprano anche importanti prospettive di approfondimento: a cui ho poco da aggiungere, se non per affermare che le sottoscrivo in toto. Vorrei solo rilevare un aspetto, che mi pare emerga con nettezza: il carattere di snodo, di crocevia, di luogo per eccellenza di intersezione fra privato e pubblico, fra Stato e società, fra individuale e collettivo come tratto caratterizzante la storia del lavoro, in particolare in età

7. Ho svolto qualche osservazione sul punto in erasi (2018).

8. Per l'importanza della traduzione di Lavoro, cultura e mentalità nella società industriale di Eric Hobsbawm vedi Bidussa (1998); Agosti (2011).

9. Musso (2011), p. 8.

10. Gozzini (1990); Id. (1991).

11. Vedi Coin (2017). 
contemporanea. Per questa ragione, l'accento che in questo volume è stato posto sul fatto normativo e istituzionale vuole porre in evidenza, nel crocevia rappresentato dal lavoro, i fattori di tensione, dissimmetria e disequilibrio che nel momento normativo trovano una proiezione e danno luogo a una composizione, che a sua volta produce riflessi nei rapporti fra la società e lo Stato ${ }^{12}$. Il dialogo a distanza fra Stolzi e Pesante, sul tema della libertà contrattuale e della servitù sostanziale, ha il merito di mostrarci l'aporia ineludibile fra il fondamento individualistico e volontaristico dei rapporti sociali e giuridici di età contemporanea, e il suo poggiare su diseguaglianze tanto concrete e stringenti da svuotarne l'esercizio, e da chiamare in causa il soggetto pubblico, lo Stato, per ridefinire indirizzo e i fini della mediazione fra i due poli. E consente anche di chiarire la scelta, nel titolo del volume, delle "libertà", al plurale, come sostantivo del lavoro: libertà al plurale vuole alludere in primo luogo al retaggio cetuale, particolaristico del rapporto di lavoro, e al prezzo ambiguo della sua emancipazione; vuole riconoscere i contorni di una "dialettica dell'individualismo" entro la quale - per richiamare l'insolubile contraddizione definita da Adorno e Horkheimer - si situa il lavoro tra libertà e servitù, diritti e mercato, fin dentro la crisi della nostra contemporaneità.

\section{Riferimenti bibliografici}

Agosti A. (2011), Il test di una vita. Profilo di Eric Hobsbawm, in "Passato e Presente", n. 82, pp. $115-140$

Artifoni E. (1990), Salvemini e il medioevo. Storici italiani fra Otto e Novecento, Napoli, Liguori.

Bidussa D. (1998), Storia e storiografia sul movimento operaio nell'Italia del secondo dopoguerra. Gli anni della formazione (1945-1956), in Il socialismo e la storia. Studi per Stefano Merli, a cura di L. Cortesi e A. Panaccione, Milano, FrancoAngeli, pp. 183-230.

Bonazza G., Ongaro G. a cura di, (2018), Libertà e coercizione. Il lavoro in una prospettiva di lungo periodo, Palermo, Ed. Sislav-NDF.

Cazzetta G. (2014), Intervento dello Stato e libertà contrattuale fra Otto e Novecento, in "Historia et ius", www.historiaetius.eu - 6- paper 2.

Cerasi L. (2018), Corporazione e lavoro. Un campo di tensione nel fascismo degli anni Trenta, in "Studi Storici", n. 4, pp. 971-992.

Cervelli I., (1977), Gioacchino Volpe, Napoli, Guida, 1977, p. 51.

Cervelli I., (1983), Gli storici italiani e l'incontro con il marxismo, in Il mondo contemporaneo, a cura di N. Tranfaglia, vol. 10, Gli strumenti della ricerca, t. 2, Questioni di metodo, Firenze, La Nuova Italia, pp. 587-614.

Coin F. (2017), La fine del lavoro (pagato), in Salari rubati. Economia politica e conflitto ai tempi del lavoro gratuito, a cura di F. Coin, Verona, Ombre Corte, pp. 7-30.

Favilli P. (1996), Storia del marxismo italiano. Dalle origini alla grande guerra, FrancoAngeli, Milano.

Favilli P. (2006), Marxismo e storia. Saggio sull'innovazione storiografica in Italia, FrancoAngeli, Milano.

Gozzini G. (1990), Lavoro e classe. Le tendenze della storiografia, in «Passato e presente», n. 24, pp. 97-111.

Gozzini G. (1991), La storiografia del movimento operaio: tra storia politica e storia sociale, in La storiografia dell'Italia contemporanea, a cura di C. Cassina, Pisa, Giardini, pp. 241-276.

Hobsbawm, E.J. (1986), Lavoro, cultura e mentalità nella società industriale, Roma-Bari, Laterza (ed. or. Worlds of Labour. Further Studies in the History of Labour, London, Weidenfled \&Nicholson, 1984).

Musso S. (2011), Storia del lavoro in Italia dall'Unità ad oggi, Venezia, Marsilio.

\section{Cazzetta (21014)}

